\title{
BANCO DE SEMENTES COMO INDICADOR DE RESTAURAÇÃO DE UMA ÁREA DEGRAdAdA POR MINERAÇÃO DE CAULIM EM BRÁS PIRES, MG ${ }^{1}$
}

Sebastião Venâncio Martins ${ }^{2}$, Diego Pierre de Almeida ${ }^{3,4}$, Loane Vaz Fernandes ${ }^{3}$ e Tiago Maciel Ribeiro ${ }^{3,4}$

\begin{abstract}
RESUMO - Este estudo teve como objetivo caracterizar o banco de sementes sob vegetação secundária em uma área degradada por mineração de caulim em Brás Pires, MG. Foram coletadas 40 amostras de solo de $0,5 \times 0,5 \mathrm{~m}$ até a profundidade de $5,0 \mathrm{~cm}$. As amostras foram mantidas em viveiro por quatro meses, sendo metade em sombreamento de $11,5 \%$ (luz) e metade em sombreamento de $60 \%$ (sombra). A germinação das sementes foi comparada nas duas condições de sombra (11,5\% e 60\%), utilizando-se o teste $t$ para amostras independentes. Foram amostradas 36 espécies pertencentes a 17 famílias botânicas. As famílias com maior riqueza específica foram Asteraceae, com nove espécies, Rubiaceae com cinco e Poaceae com quatro. A maioria das espécies $(66,7 \%)$ e dos indivíduos $(82,2 \%)$ amostrados no banco foi de herbácea. As espécies com maior número de indivíduos germinados do banco foram as herbáceas Urochloa decumbens (Stapf) R.D. Webster, Cenchrus sp. e Eragrostis sp. e a arbustiva Leandra niangaeformis Cogn. Entre as arbóreas, destacaram-se em número de indivíduos Luehea grandiflora Mart. e Trema micranta (L.) Blume. A maior densidade de ervas daninhas oriundas de áreas antropizadas do entorno indicou baixa resiliência da vegetação presente na área degradada em caso de ocorrer alguma perturbação severa.
\end{abstract}

Palavras-chave: Banco de sementes do solo, restauração de áreas degradadas e indicadores de restauração ecológica.

\section{SEED BANK AS INDICATOR OF RESTORATION OF A KAOLIN MINING - DEGRADED AREA IN BRÁS PIRES, MG}

\begin{abstract}
This study aimed to characterize the soil seed bank under secondary vegetation in an area degraded by kaolin mining in Brás Pires, MG, Brazil. Forty soil samples of $0.5 \times 0.5 \mathrm{~m}$ were collected to the depth of $5.0 \mathrm{~cm}$. The soil samples were maintained in nursery for four months, half in shading of $11.5 \%$ (light), and half in shading of 60\% (shade). Seed germination was compared under the two shade conditions (11.5\% and 60\%), utilizing the t test for independent samples. Thirty-six species belonging to 17 botanical families were sampled. The families with the largest number of species were Asteraceae with 9, Rubiaceae with 5, and Poaceae with 4. Most of the species sampled (66.7\%) were herbaceous. The most abundant species were the herbaceous Urochloa decumbens (Stapf) R.D. Webster, Cenchrus sp. and Eragrostis sp., and the shrub Leandra niangaeformis Cogn. Among the tree species, Luehea grandiflora Mart. and Trema micranta (L.) Blume presented the largest number of individuals. The largest density of the herbs originating from the disturbed surrounding areas indicates a low resilience of the vegetation in the degraded area, in case a severe disturbance occurs.
\end{abstract}

Keywords: Soil seed bank, restoration of degraded areas and ecological restoration indicators.

\footnotetext{
${ }^{1}$ Recebido em 08.11.2006 e aceito para publicação em 22.08.2008.

${ }^{2}$ Departamento de Engenharia Florestal da UFV e Bolsista Produtividade CNPq, Viçosa-MG. E-mail: <venancio@ufv.br>.

${ }^{3}$ Estudantes de Engenharia Florestal e estagiários da área de Ecologia e Restauração Florestal da UFV Viçosa-MG.

${ }^{4}$ Bolsistas de Iniciação Científica do CNPq e da FAPEMIG.
} 


\section{INTRODUÇÃO}

Atualmente, têm-se buscado alternativas para a recuperação de áreas degradadas que possibilitem a redução dos custos de recuperação e o retorno dessas áreas a uma condição ecológica mais próxima da original, ou seja, a restauração ecológica (ARONSON et al., 1995; CAIRNS JR. e HECKMAN, 1996; CHOI, 2004; YOUNG et al., 2005; GANDOLFI et al., 2006).

Dentro dessa nova tendência da recuperação de áreas têm sido preconizados o manejo e a indução dos processos ecológicos, visando aproveitar ou estimular a capacidade de auto-recuperação dos ecossistemas (resiliência). Para isso, o conhecimento das comunidades colonizadoras de áreas degradadas, bem como da autoecologia das espécies que as compõem, é fundamental para a definição de metodologias de restauração (RODRIGUES e GANDOLFI, 1998).

O banco de sementes é constituído tanto por sementes produzidas em determinada área quanto por aquelas transportadas de outros locais, sendo essas viáveis, em estado de dormência real ou imposta, presentes na superfície ou no interior do solo (HARPER, 1977; FENNER, 1985). É um componente de extrema importância na conservação de populações de plantas (HARPER, 1977; PUTZ, 1983; SWAINE e HALL, 1983; GARWOOD, 1989), participando de processos ecológicos, como o restabelecimento de comunidades após distúrbios e a manutenção da diversidade de espécies, entre outros (PUTZ, 1983; SWAINE e HALL, 1983; LAWTON e PUTZ, 1988; GARWOOD, 1989).

Nesse contexto, o banco de sementes do solo, considerando-se sua composição florística e densidade, pode ser um bom indicador para a restauração de ecossistemas (RODRIGUES e GANDOLFI, 1998; MARTINS, 2001), uma vez que, através da sua avaliação, relativamente rápida e de baixo custo financeiro, é possível definir estratégias para acelerar o processo de sucessão ecológica nas áreas em restauração.

Este estudo teve como objetivo caracterizar o banco de sementes sob vegetação secundária em uma área degradada por mineração de caulim em Brás Pires, MG, através da análise de sua composição florística e densidade de sementes, visando discutir aspectos como estado de recuperação e resiliência da vegetação nativa na área estudada.

R. Árvore, Viçosa-MG, v.32, n.6, p.1081-1088, 2008

\section{MATERIAL E MÉTODOS}

\section{1. Área de estudo}

O estudo foi desenvolvido em uma área degradada na Fazenda Boa Esperança, situada à margem esquerda do rio Turvo, Município de Brás Pires (43ํ 10' 41'long. W e $20^{\circ} 54^{\prime} 25^{\prime}$ ' lat. S), distante $60 \mathrm{~km}$ de Viçosa, MG (Figura 1). A encosta, área-alvo desta pesquisa, ocupa aproximadamente 2 ha e apresenta declividade de $35 \%$ e altitude que varia de $825 \mathrm{~m}$ no terço superior a 745 $\mathrm{m}$ no terço inferior. Conforme a classificação de Köppen, o clima da região é Cwb, apresentando verões quentes e úmidos, com pluviosidade anual de 1.200 a $1.500 \mathrm{~mm}$, estação seca curta no inverno e temperatura média anual entre $19,5^{\circ} \mathrm{C}$ e $21,8^{\circ} \mathrm{C}$ (NATALI et al., 1997). A atividade minerária foi iniciada em 1980 e finalizada em 1985. Nos 20 anos subseqüentes, a encosta onde foi depositado o estéril da mineração de caulim, anteriormente utilizada como pastagem, foi isolada, e iniciou-se um processo de regeneração natural, estando atualmente ocupada por uma vegetação florestal secundária.

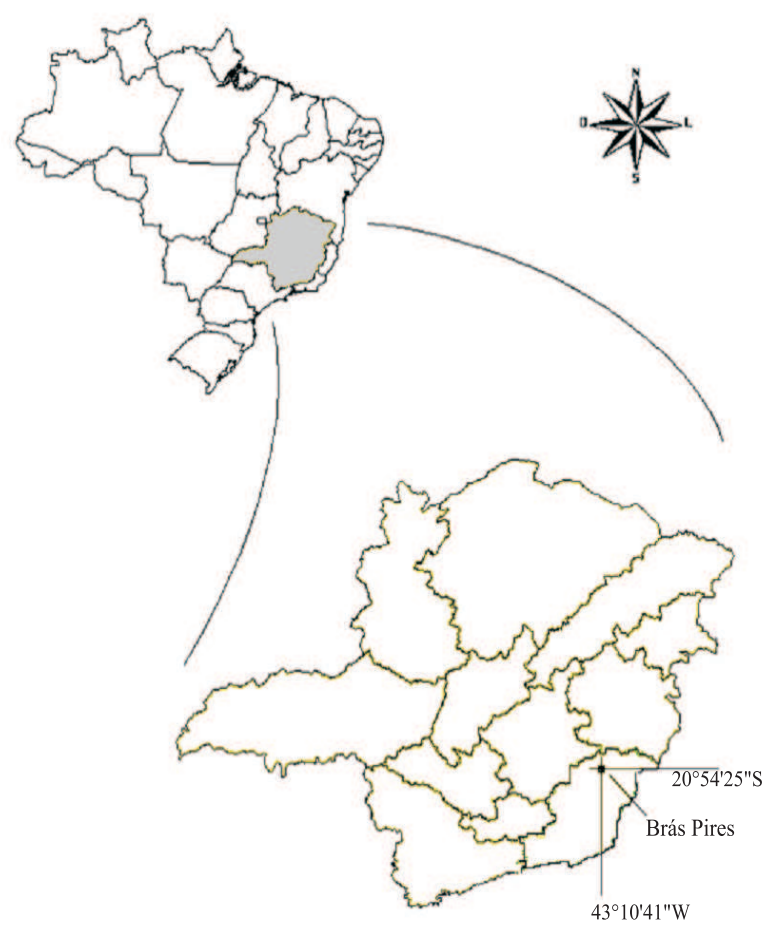

Figura 1 - Localização geográfica do Município de Brás Pires, MG

Figure 1 - Geographical Location of the Municipal district of Brás Pires, $M G$. 
Um levantamento florístico da vegetação arbustivoarbórea em regeneração na área revelou a predominância de espécies secundárias iniciais, seguidas de espécies pioneiras, caracterizando uma fase de transição da floresta pioneira para estádios mais avançados (ARAÚJO et al., 2005).

O solo da área foi caracterizado por Araújo et al. (2005), por meio de três perfis, como Latossolo VermelhoAmarelo distrófico, sendo o horizonte $\mathrm{Bw}$ pouco espesso. No topo da encosta, percebe-se a exposição do horizonte $\mathrm{C}$, devido à intensa erosão. Ao longo da encosta, pela deposição do estéril sobre o solo original, desenvolveuse novo horizonte A pouco espesso, advindo da vegetação. Nos perfis amostrados há uma diferenciação clara entre o material de origem do solo (horizonte C), o solo original (soterrado) e a camada de estéril.

A camada de estéril mostrou profundidade variável (0 a $25 \mathrm{~cm})$, sobre a qual se percebe o desenvolvimento de um horizonte $\mathrm{A}(0-2 \mathrm{~cm})$, que pode estar relacionado com a abundância de espécies pioneiras na área, favorecendo a maior produção de serapilheira e, conseqüentemente, a maior incorporação de matéria orgânica no solo (ARAÚJO et al., 2005).

Análises químicas revelaram que, de acordo com os critérios de interpretação de resultados de análises de solos propostos por Alvarez et al. (1999), a camada de estéril apresenta acidez elevada, baixa capacidade de troca catiônica (CTC) efetiva e saturação de bases muito baixa $(8,6 \%)$, caracterizando um substrato ácido e com fertilidade química muito baixa (ARAÚJO et al., 2005). Difratogramas de raios $x$ indicaram a presença de caulinita (mineral silicatado) e de goethita (óxido hidratado de ferro), o que explica a baixa CTC da camada de estéril.

\subsection{Caracterização do banco de sementes}

A amostragem do banco de sementes foi realizada numa rede de 50 parcelas contíguas de 10 x $10 \mathrm{~m}$, utilizada anteriormente para estudo florístico e fitossociológico (ARAÚJO et al., 2005). Foram sorteadas 40 dessas parcelas, nas quais foram coletadas amostras de solo. Em cada parcela sorteada foi lançada ao acaso uma moldura de $0,50 \times 0,50 \mathrm{~m}\left(0,25 \mathrm{~m}^{2}\right)$, totalizando $10 \mathrm{~m}^{2}$ nas 40 amostras. No interior dessa moldura foi coletado o solo superficial até uma profundidade de $5 \mathrm{~cm}$.

A coleta das amostras de solo foi realizada no final de setembro, época de transição entre estações seca e chuvosa, visando coincidir com o período de maior produção de frutos e sementes, verificado em Floresta Semidecídua da região (PINTO, 2005), e favorecer a germinação de maior número de espécies.

As amostras foram transportadas ao viveiro da Universidade Federal de Viçosa e colocadas em 40 caixas de madeira de 50 × $50 \mathrm{~cm}$ e $10 \mathrm{~cm}$ de altura. Metade das amostras (20 caixas) foi disposta sobre bancada coberta com tela de náilon transparente, com sombreamento de $11,5 \%$, e a outra foi mantida sobre bancada coberta com tela de náilon com sombreamento de $60 \%$. Nas duas condições de sombreamento foram mantidas cinco caixas preenchidas com areia grossa esterilizada, para verificação da possibilidade de contaminação com sementes oriundas das áreas do entorno. Todas as amostras receberam quatro irrigações diárias no decorrer do experimento. Mensalmente, durante um período de quatro meses foram realizadas a contagem e identificação das plântulas oriundas do banco de sementes do solo.

\section{RESULTADOS E DISCUSSÃO}

A densidade média de sementes germinadas por caixa $(0,5 \times 0,5 \mathrm{~m})$ foi de $203,75 \pm 54,82$ na condição de luz (sob tela com sombreamento de $11,5 \%$ ) e de $225,05 \pm 76,07$ nas caixas mantidas na sombra (sob tela com sombreamento de $60 \%$ ), contudo essas médias não diferiram estatisticamente pelo de Teste $t$ nas amostras independentes $(P>0,05)$. Nas caixas-controle com areia não foram observadas sementes germinadas durante o período do estudo. Isso indica que em estudos de avaliação do banco de sementes as amostras de solo devem ser mantidas cobertas com tela de náilon de $11,5 \%$ a $60 \%$ de sombreamento, como medida para evitar a contaminação por propágulos externos por meio de dispersão pelo vento e, ou, animais, que tende a ocorrer quando as amostras são mantidas a pleno sol.

A densidade do banco de sementes varia muito de um local para outro, mas tende a ser maior em florestas secundárias, nas quais o dossel mais aberto possibilita maior densidade de espécies pioneiras, cujas sementes possuem dormência (GARWOOD, 1989; GORRESIOROIZMAN, 1993; BAIDER et al., 2000, 2001; DALLING, 2002). Considerando-se as duas situações de sombreamento, a densidade média de sementes do banco do solo foi de $857,6 \mathrm{sementes} / \mathrm{m}^{2}$, valor intermediário aos de densidade média indicados por Garwood (1989) em florestas tropicais secundárias e primárias, de 1.650

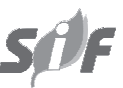

R. Árvore, Viçosa-MG, v.32, n.6, p.1081-1088, 2008 
sementes $/ \mathrm{m}^{2}$ e 380 sementes $/ \mathrm{m}^{2}$, respectivamente, e próximo ao valor de 872 sementes $/ \mathrm{m}^{2}$ estimado em trecho de Floresta Atlântica no Parque Estadual de Intervales, SP (BAIDER et al., 1999). Contudo, como indicador de avaliação de restauração, o banco de sementes deve ser analisado quanto à distribuição da sua densidade entre espécies de diferentes formas de vida.

$\mathrm{Na}$ Tabela 1 são apresentados os resultados da germinação de espécies vegetais oriundas do banco de sementes coletado no substrato superficial sob a vegetação arbustivo-arbórea colonizadora da área degradada.

Foram amostradas 36 espécies pertencentes a 17 famílias botânicas. As famílias com maior riqueza específica foram Asteraceae, com nove espécies, Rubiaceae com cinco e Poaceae com quatro. A maioria das espécies e dos indivíduos amostrados no banco é herbácea (Figura 2). Apenas oito espécies são arbóreas: Luehea grandiflora, Trema micrantha, Tibouchina granulosa, Vernonanthura diffusa, Schinus terebenthifolius, Dalbergia nigra, Machaerium acutifolium e Platypodium elegans.

Tabela 1 - Espécies germinadas no banco de sementes de área degradada por mineração de caulim em Brás Pires, MG, Brasil, e respectivas formas de vida

Table 1-Germinated species in the seed bank of area degraded by kaolin mining in Brás Pires, MG, Brazil, and their respective life forms

\begin{tabular}{|c|c|c|c|}
\hline \multirow[t]{2}{*}{ Família/Espécie } & \multirow[t]{2}{*}{ Formas de vida } & \multicolumn{2}{|c|}{ Número de indivíduos } \\
\hline & & Luz & Sombra \\
\hline \multicolumn{4}{|l|}{$\overline{\text { Anacardiaceae }}$} \\
\hline Schinus terebinthifolius Raddi & Árvore & 1 & - \\
\hline \multicolumn{4}{|l|}{ Aristolochiaceae } \\
\hline Aristolochia sp. & Trepadeira & 6 & 11 \\
\hline \multicolumn{4}{|l|}{ Asteraceae } \\
\hline Ageratum conyzoides $\mathrm{L}$. & Erva & 4 & 3 \\
\hline Baccharis trimera (Less.) DC. & Erva & 35 & 97 \\
\hline Blainvillea sp. & Erva & 41 & 5 \\
\hline Erechtites hieraciifolius (L.) Raf. ex DC. & Erva & 2 & 1 \\
\hline Eupatoruim sp. & Erva & 18 & 14 \\
\hline Gnaphalium spicatum Mill. & Erva & 22 & 20 \\
\hline Sonchus sp. & Erva & 4 & 2 \\
\hline Vernonanthura diffusa (Less.) H. Rob. & Árvore & 12 & 23 \\
\hline Vernonia polyanthes Less & Arbusto & - & 5 \\
\hline \multicolumn{4}{|l|}{ Dennstaedtiaceae } \\
\hline $\begin{array}{l}\text { Pteridium aquilinum (L.) Kuhn } \\
\text { Pneraceae }\end{array}$ & Erva & 5 & 6 \\
\hline \multicolumn{4}{|l|}{ Cyperaceae } \\
\hline \multicolumn{4}{|l|}{ Euphorbiaceae } \\
\hline \multicolumn{4}{|l|}{ Lamiaceae } \\
\hline Peltodon radicans Pohl & Erva & - & 3 \\
\hline \multicolumn{4}{|l|}{ Leguminosae - Papilionoideae } \\
\hline Dalbergia nigra (Vell.) Allemao ex Benth. & Árvore & 1 & - \\
\hline Machaerium acutifolium Vogel & Árvore & - & 1 \\
\hline Platypodium elegans Vogel & Árvore & 1 & - \\
\hline \multicolumn{4}{|l|}{ Malvaceae } \\
\hline Sida sp. & Erva & 12 & - \\
\hline \multicolumn{4}{|l|}{ Melastomataceae } \\
\hline Leandra niangaeformis Cogn. & Arbusto & 184 & 625 \\
\hline Tibouchina granulosa (Desr.) Cogn. & Árvore & 43 & - \\
\hline \multicolumn{4}{|l|}{ Oxalidaceae } \\
\hline Oxalis corniculata $\mathrm{L}$. & Erva & 54 & 64 \\
\hline
\end{tabular}

R. Árvore, Viçosa-MG, v.32, n.6, p.1081-1088, 2008 
Tabela 1 - Cont

Table 1 - Cont.

\begin{tabular}{|c|c|c|c|}
\hline \multirow[t]{2}{*}{ Família/Espécie } & \multirow[t]{2}{*}{ Formas de vida } & \multicolumn{2}{|c|}{ Número de indivíduos } \\
\hline & & Luz & Sombra \\
\hline \multicolumn{4}{|l|}{ Poaceae } \\
\hline Urochloa decumbens (Stapf) R.D. Webster & Erva & 1416 & 1855 \\
\hline Cenchrus sp. & Erva & 745 & 957 \\
\hline Echinochloa sp. & Erva & 17 & 17 \\
\hline Eragrostis $\mathrm{sp}$ & Erva & 725 & 264 \\
\hline \multicolumn{4}{|l|}{ Poligalaceae } \\
\hline Polygala paniculata $\mathrm{L}$. & Erva & 10 & - \\
\hline \multicolumn{4}{|l|}{ Rubiaceae } \\
\hline Diodia alata Nees \& Mart. & Erva & 11 & 61 \\
\hline Rubiaceae 1 & Erva & 12 & 63 \\
\hline Rubiaceae 2 & Erva & 51 & 69 \\
\hline Rubiaceae 3 & Erva & 6 & 54 \\
\hline Spermacoce latifolia Aubl. & Erva & 61 & 74 \\
\hline \multicolumn{4}{|l|}{ Solanaceae } \\
\hline Solanum sp. & Erva & - & 1 \\
\hline \multicolumn{4}{|l|}{ Tiliaceae } \\
\hline Luehea grandiflora Mart. & Árvore & 405 & 84 \\
\hline Triumfetta bartramia $\mathrm{L}$. & Arbusto & 16 & 3 \\
\hline \multicolumn{4}{|l|}{ Ulmaceae } \\
\hline Trema micranta (L.) Blume & Árvore & 57 & 43 \\
\hline
\end{tabular}

Luz = amostras de solo mantidas sob sombra de $11,5 \%$; sombra = amostras mantidas sob sombra de $60 \%$. Light $=$ soil samples maintained under shade of 11,5\%; Shade = soil samples maintained under shade of $60 \%$.

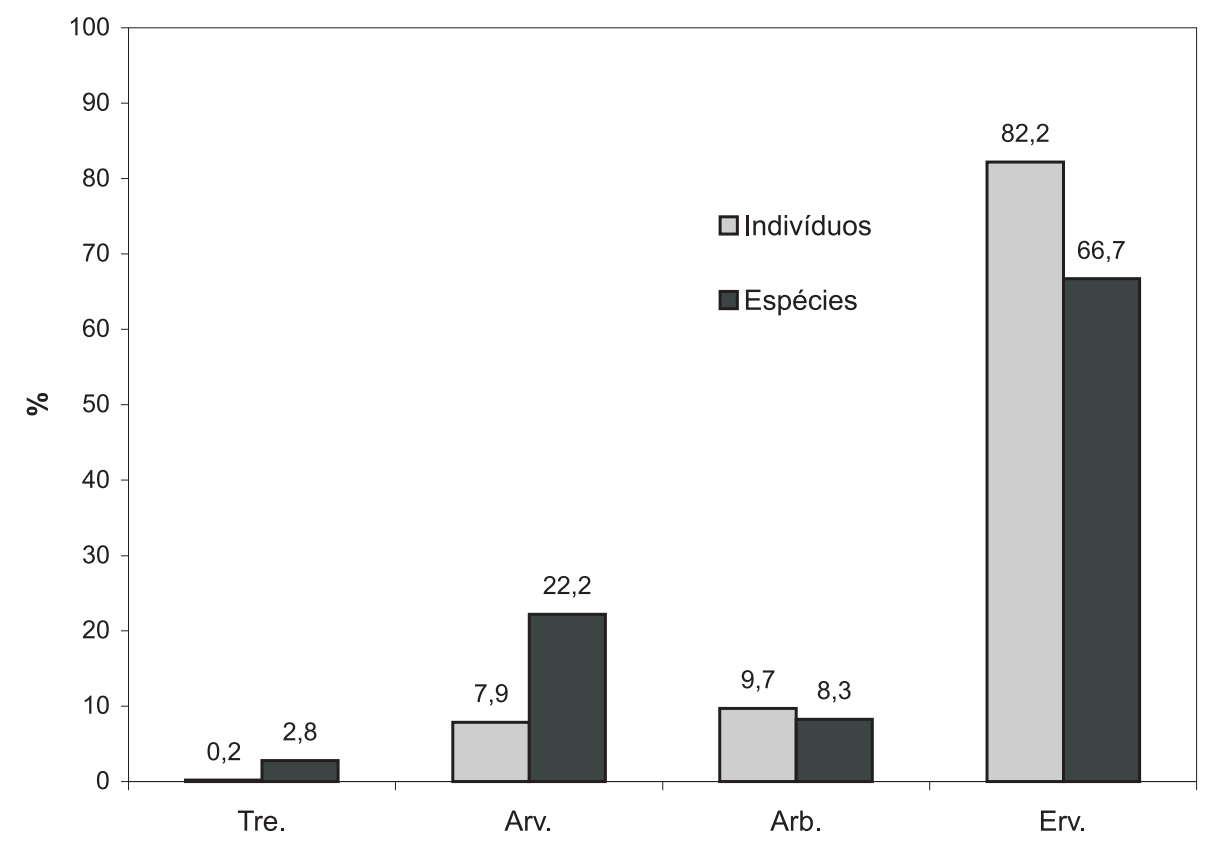

Figura 2 - Porcentagens de espécies e indivíduos, por forma de vida, amostradas no banco de sementes em uma área degradada por mineração de caulim em Brás Pires, MG, Brasil. Ter. = trepadeira; Arv. = árvore; Arb. = arbusto; e Erv. = erva.

Figure 2 - Percentages of species and individuals by life forms sampled in the seed bank in an area degraded by kaolin mining in Brás Pires, MG. Brazil. Tre. = climber; Arv. = tree; Arb. = shrub; Erv. = herb. 
Entre as espécies arbóreas, destacaram-se em número de indivíduos Luehea grandiflora e Trema micrantha. A elevada densidade de sementes germinadas de Luehea grandiflora pode ser explicada pelo fato de ser a espécie com maior número de indivíduos em levantamento florístico e fitossociológico realizado nessa área (ARAÚJO et al., 2005). Já Trema micrantha apresenta apenas um indivíduo adulto na comunidade, mas tem eficiente dispersão por pássaros e grande longevidade das sementes no solo, o que tem favorecido sua abundância no banco de sementes em várias florestas (GROMBONE-GUARATINI e RODRIGUES, 2002; NAVE, 2005). Essa espécie tem sido encontrada colonizando rapidamente áreas florestais perturbadas, como bordas e grandes clareiras (BROKAW, 1985, 1987; CASTELLANI S STUBBLEBINE, 1993; MARTINS e RODRIGUES, 2002; MARTINS et al., 2004) e uma área degradada por mineração de ouro em Matupá, MT (RODRIGUES et al., 2004).

Cabe destacar que, apesar de ser uma das espécies arbóreas mais abundantes no banco, a densidade de Trema micrantha é extremamente baixa se comparada com a de várias espécies de plantas daninhas também agressivas (Tabela 1), e, portanto, sua capacidade de colonização da área diante de um eventual distúrbio severo, como fogo, possivelmente seria inibida por essas plantas.

As espécies com maior número de indivíduos germinados do banco foram as herbáceas daninhas Urochloa decumbens, Cenchrus sp. e Eragrostis sp. e a arbustiva Leandra niangaeformis. Urochloa decumbens tem sido amostrada no banco de sementes do solo em áreas florestais degradadas exercendo forte competição com espécies arbóreas nativas (NAVE, 2005).

De acordo com Martins (2001), em projeto de restauração florestal em área degradada um banco de sementes do solo rico em espécies de plantas daninhas ou ruderais sugere que, diante de um distúrbio natural ou antrópico, com abertura de clareiras, essas espécies poderão vir a colonizar a área e competir e inibir as espécies autóctones, afetando a sustentabilidade do ecossistema. Portanto, o banco de sementes da área estudada, composto principalmente por ervas daninhas oriundas de áreas antropizadas do entorno, evidencia uma possível fragilidade da vegetação arbustivo-arbórea presente na área degradada caso ocorra alguma perturbação severa.

R. Árvore, Viçosa-MG, v.32, n.6, p.1081-1088, 2008
Vale ressaltar que existe tendência de redução na densidade de espécies herbáceas no banco de sementes e aumento de arbóreas com o avanço na sucessão em florestas secundárias (LEAL FILHO, 1992; BAIDER et al., 2001; DALLING, 2002). Dessa forma, espera-se que, mantida a situação atual de isolamento dos principais fatores de degradação, entrada de gado bovino e ocorrência de fogo, o banco de sementes seja enriquecido com sementes de espécies arbóreas oriundas de remanescentes florestais existentes na propriedade, o que aumentaria sua importância na resiliência e dinâmica do ecossistema, através do estabelecimento de populações e grupos ecológicos, restauração e manutenção da riqueza de espécies (PUTZ e APPANAH, 1987; GARWOOD, 1989; BAIDER et al., 1999; DALLING, 2002), processo que pode ser acelerado através da semeadura direta de espécies arbóreas nativas.

\section{AGRADECIMENTOS}

À Fundação de Amparo à Pesquisa do Estado de Minas Gerais - FAPEMIG, pelo financiamento do projeto (EDT - 2186/2003) e pela bolsa de Iniciação Científica (PROBIC) de T. M. Ribeiro; e ao Conselho Nacional de Desenvolvimento Científico e Tecnológico-CNPq, pelas bolsas de Produtividade em Pesquisa de S. V. Martins e de Iniciação Científica (PIBIC) de D.P. de Almeida.

\section{REFERÊNCIAS}

ALVAREZ V., V. H. et al. Interpretação dos resultados das análises de solos. In: RIBEIRO, A. C.; GUIMARÃES, P. T. G.; ALVAREZ V., V. H. (Eds.). Recomendações para uso de corretivos e fertilizantes em Minas Gerais. Viçosa, MG: CFSEMG. 1999. p.25-32.

ARAÚJO, F. S. et al. Florística da vegetação arbustivo-arbórea colonizadora de uma área degradada por mineração de caulim, Brás Pires, MG. Revista Árvore, v.29, n.6, p.107-116, 2005.

ARONSON, J. et al. Restauration et rehabilitation des ecosystems degrades en zones arides et semiarides. Lê vocabulaire et lês concepts. In: PONTANIER, C. et al. (Eds.) L' homme peutil refaire ce qu'il a défait? Paris: John Libbey Eurotext, 1995. p.11-29.

BAIDER, C.; TABARELLI, M.; MANTOVANI, W. O banco de sementes de um trecho de uma Floresta Atlântica Montana (São Paulo - Brasil). Revista Brasileira de Biologia, v.59, n.2, p.319-328, 1999.

ore, Viçosa-MG, v.32, n.6, p.1081-1088, 2008 
BAIDER, C.; TABARELLI, M.; MANTOVANI, W. The soil seed bank during Atlantic forest regeneration in Southeast Brazil. Revista Brasileira de Biologia, v.61, n.1, p.35-44, 2001.

BROKAW, N. V. L. Gap-phase regeneration in a tropical forest. Ecology, v.66, n.3, p.682-687, 1985.

BROKAW, N. V. L. Gap-phase regeneration of three pioneer tree species in a tropical forest. Journal of Ecology, v.75, n.1, p.9-19, 1987.

CAIRNS JR., J.; HECKMAN, J. R. Restoration ecology: the state of an emerging field. Annual Review of Energy and Environment, v.21, p.167-189, 1996.

CASTELLANI, T. T.; STUBBLEBINE, W. H. Sucessão secundária inicial em mata tropical mesófila, após perturbação por fogo. Revista Brasileira de Botânica, v.16, n.2, p.181-203, 1993.

CHOI, Y. D. Theories for ecological restoration in changing environment: Toward "futuristic" restoration. Ecological Research, v.19, n.1, p.75-81, 2004.

DALLING, J. W. Ecología de semillas. In: GUARIGUATA, M. R.; KATTAN, G. H. (Eds.). Ecología y conservación de bosques neotropicales. Cartago: Libro Universitario Regional, 2002. p.345-375.

FENNER, M. Seed ecology. London: Chapman and Hall, 1985. 151p.

GANDOLFI, S.; MARTINS, S. V.; RODRIGUES, R. R. Forest restoration. In: RODRIGUES, R. R.; MARTINS, S. V.; GANDOLFI, S. (Eds.) High diversity forest restoration in degraded areas: methods and projects in Brazil. New York: Nova Science Publishers, 2006.

GARWOOD, N. C. Tropical soil seed banks: a review. In: LECK, M. A.; PARKER, V. T.; SIMPSON, R. L. (Eds.) Ecology of soil seed banks. San Diego: Academic Press, 1989. p.149-209.

GORRESIO-ROIZMAN, L. G. Fitossociologia e dinâmica do banco de sementes de populações arbóreas de floresta secundária em São Paulo, SP. 1993. $184 \mathrm{f}$. Dissertação (Mestrado em Ecologia) Universidade de São Paulo, São Paulo, 1993.
GROMBONE-GUARATINI, M. T.; RODRIGUES, R. $\mathrm{R}$. Seed bank and seed rain in a seasonal semideciduous forest in south-eastern Brazil. Journal of Tropical Ecology, v.18, n.5, p.759-774, 2002.

HARPER, J. L. Population biology of plants. London: Academic Press, 1977. 892p.

LAWTON, R. O.; PUTZ, F. E. Natural disturbance gap-phase in a wind-exposed tropical sloud forest. Ecology, v.69, n.3, p.764-777, 1988.

LEAL FILHO, N. Caracterização do banco de sementes de três estádios de uma sucessão vegetal na Zona da Mata de Minas Gerais. 1992. 116f. Dissertação (Mestrado em Ciência Florestal) - Universidade Federal de Viçosa, Viçosa, MG, 1992.

MARTINS, S. V. Recuperação de matas ciliares. Viçosa, MG: Aprenda Fácil, 2001. 146p.

MARTINS, S. V.; RODRIGUES, R. R. Gap-phase regeneration in a semideciduous mesophytic forest, south-eastern Brazil. Plant Ecology, v.163, n.1, p.51-62, 2002.

MARTINS, S. V. et al. Colonization of gaps produced by death of bamboo clumps in a semideciduous mesophytic forest in southeastern Brazil. Plant Ecology, v.172, n.1, p.121-131, 2004.

NATALI, P. P.; VIANA, R. F.; FORTES, V. M. Proposta para recuperação ambiental da área de cava e adjacências da mineração de caulim na fazenda Boa Esperança, em Brás Pires - MG. In: SIMPÓSIO NACIONAL DE RECUPERAÇÃO DE ÁREAS DEGRADADAS, 3., 1997, Ouro Preto. Anais... Viçosa, MG: Universidade Federal de Viçosa, 1997.p.214-224.

NAVE, A. G. Banco de sementes autóctone e alóctone, resgate de plantas e plantio de vegetação nativa na Fazenda Intermontes, município de Ribeirão Grande, SP. 2005. 218f. Tese (Doutorado em Recursos Florestais) - Escola Superior de Agricultura Luiz de Queiroz, Piracicaba, 2005.

R. Árvore, Viçosa-MG, v.32, n.6, p.1081-1088, 2008 
PETERSEN, D. A.; CLARY, J. J. The ecology of restoration: historical links, emerging issues and unexplored realms. Ecology Letters, v.8, n.6, p.662-673, 2005.

PINTO, S. I. C. Florística, estrutura e ciclagem de nutrientes em dois trechos de Floresta Estacional Semidecidual da Reserva Florestal Mata do Paraíso, Viçosa, MG. 2005. 218f. Dissertação (Mestrado em Ciências Florestais) - Universidade Federal de Viçosa, Viçosa, MG, 2005.

PUTZ, F. E. Treefall pits and mounds, buried seeds, and the importance of soil disturbance to pioneer trees on Barro Colorado Island, Panama. Ecology, v.64, n.5, p.1069-1074, 1983.

PUTZ, F. E.; APPANAH, S. Buried seeds, newly dispersed seeds, and the dynamics of a lowland forest in Malaysia. Biotropica, v.19, n.4, p.326-333, 1987.
RODRIGUES, R. R.; GANDOLFI, S. Restauração de florestas tropicais: subsídios para uma definição metodológica e indicadores de avaliação e monitoramento. In: DIAS, L. E.; MELLO. J. W. (Eds.). Recuperação de áreas degradadas. Viçosa, MG: Universidade Federal de Viçosa; SOBRADE, 1998. p.203-215.

RODRIGUESS, R. R.; MARTINS, S. V.; BARROS, L. C. Tropical Rain Forest regeneration in an area degraded by mining in Mato Grosso State, Brazil. Forest Ecology and Management, v.190, n.2/3, p.323-333, 2004.

SWAINE, M. D.; HALL, J. B. Early succession on cleared forest land in Ghana. Journal of Ecology, v.71, n.2, p.601-627, 1983.

YOUNG, T. P.; PETERSEN, D. A.; CLARY, J. J. The ecology of restoration: historical links, emerging issues and unexplored realms. Ecology Letters, v.8, n.6, p.662-673, 2005. 